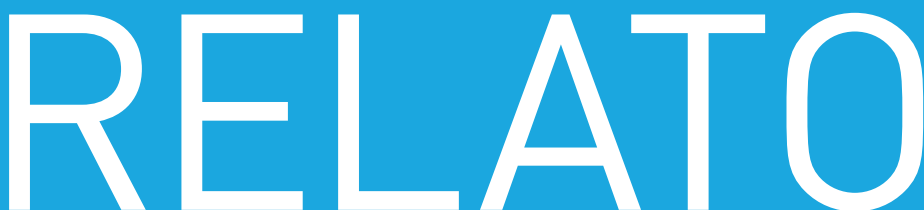

\title{
PERÍCIA PSIQUIÁTRICA ADMINISTRATIVA E TEPT: UM RELATO DE CASO
}

\section{ADMINISTRATIVE PSYCHIATRIC EVALUATION AND PTSD: A CASE REPORT}

\section{Resumo}

O presente artigo relata um caso de transtorno de estresse pós-traumático que foi submetido a uma perícia psiquiátrica administrativa. Foi concluído que, pela gravidade do caso, havia incapacidade laborativa. A perícia psiquiátrica de natureza administrativa é de extrema importância para a garantia dos direitos dos indivíduos. O estudo detalhado do caso, a coleta de anamnese completa e o exame psicopatológico são fundamentais para a sua adequada realização.

Palavras-chave: Avaliação, incapacidade, transtornos psiquiátricos.

\section{Abstract}

This article describes a case of posttraumatic stress disorder that underwent an administrative psychiatric evaluation. It was concluded that, due to the severity of the case, there was work disability. Administrative psychiatric evaluations are extremely important for guaranteeing the rights of individuals. Detailed study of the case, comprehensive anamnesis, and psychopathological examination are all fundamental to ensure that these evaluations are carried out correctly.

Keywords: Evaluation, disability, psychiatric disorders.

\section{INTRODUÇÃO}

O transtorno de estresse pós-traumático (TEPT) é marcado por aumento de estresse e ansiedade após exposição a um evento traumático ou estressante. Esses eventos podem incluir: ser testemunha ou estar envolvido em um acidente ou crime violento, agressão, ser sequestrado, estar envolvido em um desastre natural, vivenciar abuso físico ou sexual. A pessoa reage à experiência com medo e impotência, revive persistentemente o acontecido e tenta evitar lembrar dele. O evento pode ser revivido em sonhos e em pensamentos quando acordado, isto é, em flashbacks ${ }^{1}$.

O TEPT é o único quadro psiquiátrico com um evento externo definido como parte de seu diagnóstico. Uma situação que coloca em risco a integridade física ou a vida da pessoa desencadeará uma reação do organismo para que consiga sobreviver a essa ameaça. A maior parte das pessoas supera essa ameaça, mas algumas não, e esse evento se torna uma memória traumática revivida com grande sofrimento ${ }^{2}$.

Pesquisas demonstram que entre 40 e $60 \%$ das pessoas já foram expostas a eventos traumáticos, mas apenas 8\% têm TEPT. As mulheres têm duas vezes mais chances de ter o TEPT em comparação aos homens (10\% contra 5\%), apesar de os homens serem mais propensos a se exporem ao trauma (60\% de homens contra 51\% de mulheres passam por eventos traumáticos em algum momento de suas vidas) ${ }^{3}$.

Os estressores relacionados ao TEPT são suficientemente devastadores para afetar quase todas as pessoas. Estas revivem o evento traumático em seus sonhos e em seus pensamentos diários; são persistentes em evitar qualquer coisa que o traga à mente e experimentam um entorpecimento da responsividade, junto com um estado de hipervigilância. Outros sintomas são depressão, ansiedade e dificuldades cognitivas, como falta de concentração ${ }^{4}$.

Entende-se que as situações que teriam o maior potencial para o desenvolvimento de transtornos mentais 
são aquelas que provocam ruptura na estrutura social, na forma de catástrofes, ou, ainda, aquelas provocadas por outros indivíduos de maneira intencional, através de atos não condizentes ou disformes em relação à própria cultura, os chamados "atos bárbaros", como ações de bandidos em assaltos, sequestros, estupros e tentativas de homicídio 5 .

Diversos estudos têm sido realizados mundialmente e no Brasil para detectar a taxa de vitimização da população ${ }^{6}$. Estudo de Andreoli et al. ${ }^{7}$ evidenciou que, no Brasil, mais da metade dos habitantes das cidades do Rio de Janeiro e São Paulo relataram ter vivenciado um evento traumático ao longo de suas vidas. Outra pesquisa com amostra brasileira verificou que 36\% das mortes ocasionadas por eventos externos são decorrentes de homicídio ${ }^{8}$

O objetivo do presente artigo é descrever o caso de uma paciente com diagnóstico de TEPT, que foi submetida a uma perícia administrativa para avaliação de capacidade laborativa.

\section{RELATO DE CASO}

Identificação

Ana, 54 anos, natural do Nordeste, com $3^{\circ}$ grau completo de escolaridade, casada, funcionária pública.

\section{Queixa principal}

"Eu tenho muito medo de sair de casa".

\section{História da doença atual}

Refere a pericianda que, no ano de 2015, foi atingida por dois disparos de projéteis de arma de fogo, em uma tentativa de homicídio, que atingiram sua face e pescoço (sic). Esses fatos aconteceram nas proximidades da sua casa, ao parar para comprar pães em uma padaria. Afirma que, na ocasião, atuava como fiscal de serviço público. Em virtude de lesões que sofreu no rosto, foi submetida a procedimentos cirúrgicos. Nessa ocasião, permaneceu uma semana internada em hospital clínico (sic). Acredita que a motivação pela qual sofreu a agressão foi o fato de ter fiscalizado "empresas fantasmas" no exercício do seu trabalho e que foi vítima de uma possível retaliação, em virtude de suas atividades profissionais. Há 3 anos, não frequenta o local de trabalho, em virtude de intensos sintomas de ansiedade relacionados ao evento traumático.

Após o ocorrido, a pericianda afirma que esteve poucas vezes no município em que trabalhava, apenas com a finalidade de ser submetida a perícias médicas, escoltada por policiais, em virtude de apresentar "terror e medo horrível", decorrentes da situação pela qual passou. Verbaliza que, após o evento traumático, ficou "escondida" na casa de um parente, por um período de 2 meses.

A partir do ano de 2015, a pericianda refere que passou a fazer acompanhamento psiquiátrico, com uso de diversos psicofármacos antidepressivos e ansiolíticos. Esse acompanhamento persiste até os dias atuais, apresentando diversos atestados médicos e receitas de medicamentos durante o presente exame.

Relata ainda a pericianda que, após o ocorrido (tentativa de homicídio), passou a apresentar constantes pesadelos, sempre relacionados à situação de agressão: "sentia e sinto muito medo que eles voltem". Diz que é frequente, desde o ocorrido, ter pensamentos e ideias que the "invadem a mente", relacionados às cenas do trauma. Sempre sentiu-se muito assustada ao ter que andar pelas ruas, acreditando que estava sendo olhada por todos, com medo de que alguém se aproximasse e Ihe fizesse algum mal. Diz também não gostar de falar sobre esse tema, afirmando: "quando falava sobre isso, começava a chorar".

O temor de sair de casa persiste até os dias atuais, relatando que o faz apenas se estiver acompanhada de familiares: "tenho medo de que eles possam voltar". Nunca sai de casa à noite. Afirma ainda que tem pesadelos recorrentes, várias vezes por semana, relacionados com o evento traumático, em que está "tomando outro tiro".

\section{Antecedentes pessoais}

Nascida de parto normal, a termo, normal desenvolvimento neuropsicomotor. Nega viroses comuns da infância. Traumatismo facial relacionado à agressão de que foi vítima. Nega tabagismo, nega uso atual ou prévio de álcool ou substâncias psicoativas. Afirma que antes do evento traumático era uma pessoa saudável e produtiva. Faz tratamento psiquiátrico desde o ano de 2015. É hipertensa, em tratamento clínico. 


\section{Antecedentes familiares e psicossociais}

Pai falecido de infarto. Mãe falecida de câncer de mama. Tem duas irmãs saudáveis. Nega história de transtornos mentais na família.

A pericianda refere que sempre teve um ótimo aproveitamento escolar. Entrou para a faculdade de direito aos 18 anos de idade, concluindo esse curso aos 23 anos.

Fez diversos estágios com bom aproveitamento, na graduação. É casada, tem três filhas. Refere bom relacionamento conjugal. Trabalhou em escritórios de advocacia por alguns anos. Aos 35 anos, foi aprovada em concurso de serviço público, para cargo de fiscal.

De acordo com a pericianda, antes do evento traumático (tentativa de homicídio), era uma pessoa "normal e tranquila". Afirma que todas as modificações em seu comportamento (medo, insegurança, hipersensibilidade e incapacidade) se iniciaram após o evento traumático.

Relata que fica a maior parte do tempo em casa. Quase não sai de casa em virtude de medo de ser "morta por eles, que já tentaram me matar uma vez". Nunca sai de casa à noite, em virtude desse medo. Relata ter se afastado de amigos desde a ocasião do evento traumático.

\section{Exame psiquiátrico}

Apresenta atitude colaborativa, respondendo prontamente aos dados que Ihe são formulados. Trajada adequadamente para a ocasião, com higiene pessoal mantida. Desperta, lúcida, globalmente orientada. Permanece atenta à entrevista, fala clara e bem articulada. Convidada a falar de si, afirma: "Continuo com muito medo de sair de casa... E se eles voltarem para me pegar, como já fizeram uma vez? (...) Quando saio na rua, fico cismada que pode ter alguém me seguindo". A pericianda verbaliza ideias recorrentes e pesadelos envolvendo o evento traumático. Refere ter "crise de nervos" quando pensa na possibilidade de retomar atividades laborativas no município em que exerceu essas atividades. Apresenta memórias preservadas para fatos recentes e remotos; inteligência dentro dos limites de normalidade; pensamento com características de constância, organização e continuidade; ideias sobrevalorizadas de conteúdo persecutório e de autorreferência; não há presença de delírios ou alucinações; humor muito ansioso; vontade e pragmatismo prejudicados, em virtude do estado crônico de ansiedade; consciência de morbidade presente.

\section{Discussão}

A pericianda apresenta transtorno mental na forma de TEPT [código F43.1 da $10^{a}$ edição da Classificação Internacional de Doenças (CID-10)]. A CID-10 considera as seguintes características clínicas para o diagnóstico do transtorno de estresse pós-traumático:

A) Exposição a evento ou situação estressante, de curta ou longa duração, de natureza ameaçadora ou catastrófica, a qual provavelmente causaria angústia invasiva em quase todas as pessoas.

B) Rememoração ou revivência persistente do fator estressor em flashbacks intrusivos. Memórias vividas, sonhos recorrentes e angústia em circunstâncias semelhantes ou associadas ao estressor/agressor.

C) Tentativas de evitar situações semelhantes ou associadas ao estressor/agressor.

D) Um dos seguintes aspectos ou sintomas deve estar presente:

1. Incapacidade de relembrar, parcial ou completamente, alguns aspectos do período de exposição ao agressor/estressor;

2. Sintomas persistentes de sensibilidade e excitação psicológicaaumentada, demonstrada por dois dos seguintes sintomas:
(a) Dificuldade em adormecer permanecer dormindo/insônia
(b) Irritabilidade ou explosões de raiva
(c) Dificuldade de concentração
(d) Hipervigilância
(e) Resposta ao susto exagerada

E) Os critérios B, C, D devem ter a duração de 30 dias e ter todos ocorrido dentro de 6 meses do evento estressante.

No caso da pericianda, a mesma apresenta todos os critérios para esse diagnóstico. Vejamos:

Critério A: a pericianda sofreu forte evento traumático. Foi abordada por indivíduos com arma de fogo que vieram a disparar contra a sua face e pescoço, produzindo ferimentos graves ameaçadores à sua vida. 
Critério B: a pericianda apresenta pensamentos recorrentes, aversivos e intrusivos (flashbacks), e pesadelos relacionados ao evento, já descritos.

Critério C:a periciandaapresenta comportamentos desencadeados por essas memórias, com diversos componentes de evitação de lugares (tem muito medo de retornar ao trabalho/local onde ocorreu o evento) e pessoas (afastou-se de amigos, isolamento social marcante).

Critério D: apresenta a maior parte dos sintomas descritos nesse item, como insônia, dificuldade de concentração, hipervigilância, exagero de resposta ao susto ou barulhos.

Critério E: os sintomas ocorreram após o evento e persistem até os dias atuais.

De fato, pode-se afirmar que a pericianda apresenta forma crônica do TEPT, inclusive com prognóstico desfavorável, pela quase ausência de resposta ao tratamento.

Diversos estudos da literatura têm apontado a importância do estressor desencadeador econsequências físicas, psicológicas e incapacitação funcional, laborativa e social associadas ao TEPT. Um estudo epidemiológico americano, de Pietrzak et al. ${ }^{10}$, realizado com população de 34.653 indivíduos, verificou que estressores relacionados à ameaça de vida eram os mais frequentes em indivíduos que desenvolveram TEPT.

Outros estudos ${ }^{11}$ têm encontrado que o trauma interpessoal, como no caso em questão, está associado a mais sintomas, na apresentação do TEPT, e maior duração dos sintomas, quando comparados a eventos não interpessoais (exemplo, acidente com veículo). Esse tipo de pesquisa ${ }^{12}$ tem apontado não só uma maior intensidade do TEPT, após eventos traumáticos intencionais, como também maior gravidade de curso (crônico) e prognóstico, como no caso em tela.

Há ampla evidência de que vítimas de crimes graves ${ }^{13}$ e intencionais ${ }^{14}$, como no caso em questão, apresentam mais consequências emocionais e físicas, que podem levar a sentimentos de depressão e ansiedade e temor de ser novamente vítima de crimes, como acontece com a pericianda. Ora, o estressor no caso em questão foi muito grave e quase letal. A pericianda foi atacada por indivíduos e atingida por projéteis de arma de fogo em sua face, permanecendo 1 semana internada em hospital clínico. Pesquisas ${ }^{15}$ têm mostrado que mesmo um "trauma indireto" (por exemplo, ver ou saber que alguém foi atacado) pode desencadear um quadro de TEPT. Não é difícil imaginar que um trauma grave, como aquele sofrido pela pericianda, seja um desencadeador muito mais intenso para o surgimento desse transtorno mental.

Um tema importante de pesquisa na atualidade é o estudo do impacto de eventos traumáticos na qualidade de vida de indivíduos que passaram por esses eventos. Um estudo europeu ${ }^{16}$ verificou que, após passados 2 anos do evento traumático, esses indivíduos continuavam a apresentar mau funcionamento emocional, déficit funcional, déficits socioeconômicos e grande prejuízo da qualidade de vida. Não podemos deixar de reconhecer que, no caso da pericianda, todos esses aspectos estão presentes. A pericianda, antes uma pessoa produtiva e saudável, tendo ocupado várias funções importantes em sua trajetória profissional, quase não sai de casa após o evento traumático, em virtude do medo de repetição de outra agressão. Afastou-se dos amigos (isolamento social), sente-se ansiosa a maior parte do tempo, tem muita dificuldade de concentração e prejuízo cognitivo, além do grande sofrimento psíquico desde o evento traumático. Certamente, todos esses aspectos levaram a um grande prejuízo em sua qualidade de vida.

Embora a simulação seja um diagnóstico diferencial de casos de TEPT ${ }^{17}$, não há nenhuma possibilidade de que esse comportamento esteja acontecendo no caso em tela. A pericianda apresentou farta documentação médica em geral, bem como psiquiátrica. Os sintomas relatados e aferidos foram avaliados minuciosamente. A evolução do transtorno mental (TEPT) tem uma linearidade e uma coerência que traduzem a clínica real do mesmo.

Concluímos que os sintomas do transtorno mental apresentados pela pericianda (TEPT) são intensos e permanentes, levando a um grande nível de sofrimento subjetivo, prejuízo da qualidade de vida em seus diversos domínios (saúde física e emocional, sensação de bemestar, autonomia, funcionamento psicológico, papel social) e importante sofrimento psíquico. O prognóstico do caso é extremamente desfavorável e o curso do seu transtorno mental é crônico. A pericianda se enquadra 
totalmente no conceito médico-legal de incapacidade laborativa18: "impossibilidade do desempenho dasfunções em consequência de alterações morfopsicofisiológicas provocadas por doença ou acidente". O transtorno mental que apresenta (TEPT) Ihe acarreta incapacidade para o exercício de atividades laborativas. A incapacidade é omniprofissional e permanente.

A perícia psiquiátrica de natureza administrativa é de extrema importância para a garantia de direitos do indivíduo. $O$ estudo detalhado do caso, a coleta de anamnese completa e o exame psicopatológico são fundamentais para a sua adequada realização.

Artigo submetido em 28/05/2020, aceito em 28/05/2020. Os autores informam não haver conflitos de interesse associados à publicação deste artigo.

Fontes de financiamento inexistentes.

Correspondência: Alexandre Valença, Rua Conde de Bonfim, 232, sala 511, Tijuca, CEP 22221-011, Rio de Janeiro, RJ. Tel.: (21) 22644394. E-mail: avalen@uol.com.br

\section{Referências}

1. de Mello AF, Valente NLM, Fiks JP, Meleiro A. Transtorno de estresse pós-traumático. In: Meleiro A. Psiquiatria. Estudos fundamentais. Rio de Janeiro: Guanabara Koogan; 2018. p. 276-88.

2. Yeh MSL, de Mello AF, de Mello MF. Transtorno de estresse pós-traumático resistente ao tratamento. In: Carvalho AF, Nardi AE, Quevedo J, org. Porto Alegre: Artmed; 2015. p. 72-84.

3. Leahy RL. "Está acontecendo de novo": transtorno de estresse pós-traumático. Capítulo 9. In: Leahy RL. Livre de ansiedade. Porto Alegre: Artmed; 2011. p. 162-82.

4. Sadock BJ, Sadock VA, Ruiz P. Transtornos relacionados a trauma e estressores. Capítulo 11. In: Sadock BJ, Sadock VA, Ruiz P. Compêndio de psiquiatria: ciência do comportamento e psiquiatria clínica. São Paulo: Artmed; 2017. p. 437-50.

5. Braga LL, Fiks JP, Mari JJ, Mello MF. The importance of the concepts of disaster, catastrophe, violence, trauma and barbarism in defining posttraumatic stress disorder in clinical practice. BMC Psychiatry. 2008;8:68.
6. Ribeiro WS, Andreoli SB, Mari JJ. Epidemiologia da violência e sua relação com os problemas de saúde mental no Brasil. In: Fiks JP, Mello MF, editores. Transtorno de estresse pós-traumático: violência, trauma e medo no Brasil. São Paulo: Atheneu; 2011. p. 129-38.

7. Andreoli SB, Ribeiro WS, Quintana MI, Guindalini C, Breen G, Blay SL, et al. Violence and posttraumatic stress disorder in Sao Paulo and Rio de Janeiro, Brazil: the protocol for an epidemiological and genetic survey. BMC Psychiatry. 2009;9:34.

8. Reichenheim ME, de Souza ER, Moraes CL, de Mello Jorge $\mathrm{MH}$, da Silva CM, de Souza Minayo MC. Violence and injuries in Brazil: the effect, progress made, and challenges ahead. Lancet. 2011;377:1962-75.

9. OrganizaçãoMundial deSaúde(OMS).Classificação de transtornos mentais e de comportamento da CID-10. Porto Alegre: Artmed; 1992.

10. Pietrzak RH, Goldstein RB, Southwick SM, Grant BF. Prevalence and Axis I comorbidity of full and partial posttraumatic stress disorder in the United States: results from wave 2 of the national epidemiologic survey on alcohol and related conditions. J Anxiety Disord. 2011;25:456-65.

11. Chapman C, Mills K, McFarlane AC, Bryant RA, Creamer M, Silove D, et al. Remission from posttraumatic stress disorder in general population. Psychol Med. 2012;42:1605-703.

12. Santiago PN, Ursano RJ, Gray CL, Pynoos RS, Spiegel D, Lewis-Fernandez R, et al. A systematic review of PTSD prevalence trajectories in DSM-5 defined trauma exposed populations: intentional and non-intentional traumatic events. Plos One. 2013;8:e59236.

13. Green DL, Pomeroy E. Crime victimization: assessing differences between violent and nonviolent experiences. Vict Offender. 2007;6376.

14. Santiago PN, Ursano RJ, Gray CL, Pynoos RS, Spiegel D, Lewis-Fernandez R, et al. A systematic review of PTSD prevalence and trajectories in DSM-5 defined trauma exposed populations: intentional and non-intentional traumatic events. Plos One. 2013;8:e59236. 


\section{ALEXANDRE MARTINS VALENCA ${ }^{1}$ D, TALVANE MARINS DE MORAES ${ }^{2}$ LISIEUX E. DE BORBA TELLES ${ }^{3}$, ANTONIO GERALDO DA SILVA 10 , ALCINA BARROS 5}

' Doutor em Psiquiatria pelo Instituto de Psiquiatria da Universidade Federal do Rio de Janeiro (IPUB-UFRJ), Rio de Janeiro, RJ. Psiquiatra forense pela ABP. Vice-coordenador, Departamento de Psiquiatria Forense, ABP. Rio de Janeiro, RJ. Professor associado, Departamento de Psiquiatria e Saúde Mental, Universidade Federal Fluminense, Niterói, RJ. Professor, Programa de Pós-Graduaça de Psiquiatria e Saúde Mental, IPUB-UFRJ, Rio de Janeiro, RJ. Coordenador, Programa de Ensino e Pesquisa em Psiquiatria Forense, IPUB-UFRJ, Rio de Janeiro, RJ. ${ }^{2}$ Livre-docente e doutor em Psiquiatria. Membro honorário. Academia Nacional de Medicina. Professor de Psiquiatria Forense, Escola da Magistratura do Tribunal de Justiça do Estado do Rio de Janeiro (EMERJ), Rio de Janeiro, RJ. Membro titular, Academia de Medicina do Rio de Janeiro, Rio de Janeiro, RJ. Doutora em Medicina pela Universidad Nacional de La Plata (UNLP). La Plata, Argentina. Psiquiatra forense pela ABP. Coordenadora, Departamento de Psiquiatria Forense, ABP. Rio de Janeiro, RJ. Professora, Departamento de Psiquiatria e Medicina Legal, Faculdade de Medicina, UFRGS, Porto Alegre, RS. Docente, Maestria en Salud Mental Forense, UNLP, La Plata, Argentina. "Doutor pela Faculdade de Medicina da Universidade do Porto, Porto, Portugal. Presidente, ABP, Rio de Janeiro, RJ, e Asociación Psiquiatrica de Amerrica Latina: (APAL), Brasilia, DF. 'Doutora em Psiquiatria e Ciencias do Comportamento pela Universidade Federal do Rio Grande do Sul (UFRGS), Porto Alegre, RS. Medica psiquiatra forense pela ABP, Rio de Janeiro, RJ, e Universidade Federal de Ciências da Saúde de Porto Alegre [UFCSPA], Porto Alegre, RS. Secretária, Departamento de Psiquiatria Forense, ABP, Rio de Janeiro, RJ. Psicoterapeuta de orientaçáa analitica pelo Centro de Estudos Luis Guedes/Mospital de Clinicas de Porto Alegre,

15. Gil S, Caspi Y. Personality traits, coping style, and perceived threat as predictors of posttraumatic stress disorder after exposure to a terrorist attack: a prospective study. Psychosom Med 2006;68:904-9.

16. Kaske S, Lefering R, Trentzsch H, Driesse A, Bouillon B, Maegele M, et al. Quality of life two years after severe trauma: a single-centre evaluation. Injury. 2014;45 Suppl 3:S100-5.
17. Knoll J, Resnick PJ. The detection of malingered post-traumatic stress disorder. Psychiatr Clin North Am. 2006;29:629-47.

18. Villani Marques CT. Perícia administrativa. In: Epipanio EB, Xavier Vilela JRP. Perícias médicas. Teoria e prática. Rio de Janeiro: Guanabara Koogan; 2009. p. 203-17. 